\title{
Lactoferrin-derived Lytic Peptide LTX-315
}

National Cancer Institute

\section{Source}

National Cancer Institute. Lactoferrin-derived Lytic Peptide LTX-315. NCI Thesaurus. Code C90547.

A peptide derived from human lactoferrin, with potential lytic and immunostimulating activities. Upon transdermal injection directly into the tumor, LTX315 may bind to the tumor cell membranes and subsequently lyse tumor cells, thereby inducing tumor cell necrosis. In turn, presentation of the tumor antigens to the immune system may induce systemic innate and adaptive immune responses mediated by anti-tumor natural killer (NK) cells, cytotoxic T lymphocytes, and natural killer T (NKT) cells. This may trigger an immune response against tumor associated antigens on tumors distant from the primary tumor. Human lactoferrin, a 692 amino acid glycoprotein, belongs to the transferrin family of metal-binding proteins. 